\title{
REFERENCES
}

1. A. B. Simon, Vanishing algebras, to appear in Trans. Amer. Math. Soc.

2. E. J. McShane, Images of sets satisfying the condition of Baire, Ann. of Math. vol. 51 (1950) pp. 380-386.

3. B. J. Pettis, On continuity and openness of homomorphisms in topological groups, Ann. of Math. vol. 52 (1950) pp. 293-308.

4. - Remarks on a theorem of E. J. McShane, Proc. Amer. Math. Soc. vol. 2 (1951) pp. 166-171.

5. Fred B. Wright, Semigroups in compact groups, Proc. Amer. Math. Soc. vol. 7 (1956) pp. 309-311.

Tulane University

\section{SIMPLE NODAL NONCOMMUTATIVE JORDAN ALGEBRAS}

\section{LOUIS A. KOKORIS}

1. Introduction. Nodal algebras were defined by R. D. Schafer [4] and have also been studied by the author $[2 ; 3]$. A noncommutative Jordan algebra is an algebra $\mathfrak{A}$ over a field $\mathfrak{F}$ satisfying (1) the flexible law $(x y) x=x(y x)$ and (2) the condition that $\mathfrak{A}^{+}$is a Jordan algebra. That is, $\mathfrak{A}^{+}$satisfies the identity $\left(x^{2} \cdot y\right) \cdot x=x^{2} \cdot(y \cdot x)$ where we have used the dot to indicate the product of $\mathfrak{A}^{+}$. The algebra $\mathfrak{A}^{+}$ is defined to be the same vector space as $\mathfrak{A}$ but with product $x \cdot y$ $=(x y+y x) / 2$ where $x y$ and $y x$ are products in $\mathfrak{A}$. Then $\mathfrak{A}$ is called nodal if it is finite dimensional, if $\mathfrak{A}$ has identity element 1 , if $\mathfrak{A}$ can be written as a vector space direct sum $\mathfrak{A}=\mathfrak{F} 1+\mathfrak{N}$ where $\mathfrak{N}$ is the subspace of nilpotent elements of $\mathfrak{A}$, and if $\mathfrak{N}$ is not a subalgebra of $\mathfrak{A}$.

Every known nodal algebra $\mathfrak{A}$ has the property that $\mathfrak{U}^{+}$is an associative algebra. The flexible algebras with $\mathfrak{A}^{+}$associative have been described in [3]. In this paper we shall prove the following theorem.

TheоReм 1. Let $\mathfrak{i}$ be a simple nodal noncommutative Jordan algebra of characteristic $\neq 2$. Then $\mathfrak{A}^{+}$is associative.

Define $\mathfrak{B}$ to be the subspace of $\mathfrak{A}$ generated by all the associators in $\mathfrak{N}^{+}$. That is, $\mathfrak{B}$ is generated by elements of the form $(x \cdot y) \cdot z-x \cdot(y \cdot z)$ with $x, y, z$ in $\mathfrak{R}$. The proof of the theorem will be made by showing that the ideal $\mathfrak{S}$ of $\mathfrak{A}$ generated by $\mathfrak{B}$ is not all of $\mathfrak{A}$ and since $\mathfrak{A}$ is simple it will follow that $\mathfrak{C}=0$ and $\mathfrak{B}=0$. This is the desired result.

The original proof was not valid when the characteristic is 3 . The author thanks Professor R. D. Schafer for suggesting a modification

Presented to the Society, January 28, 1958; received by the editors January 13 , 1958. 
which makes the proof simpler and also valid when the characteristic is 3 .

2. The proof. Let $x_{1}, x_{2}, x_{3}$, and $y$ be any elements of $\mathfrak{R}$. Since $\mathfrak{A}$ is nodal we have:

$$
x_{i} y=\lambda_{i} 1+z_{i}
$$

The proof will depend on relation (8) of Schafer's paper [4] which is

$$
\begin{aligned}
\left(x_{1} \cdot x_{2}\right) y= & \lambda_{1} x_{2}+\lambda_{2} x_{1}+x_{1} \cdot z_{2}+x_{2} \cdot z_{1} \\
& -\left(x_{1} \cdot y\right) \cdot x_{2}-\left(x_{2} \cdot y\right) \cdot x_{1}+\left(x_{1} \cdot x_{2}\right) \cdot y .
\end{aligned}
$$

In the proof it will also be necessary to use the fact that $\mathfrak{R}^{+}$is a subalgebra of $\mathfrak{A}^{+}[1]$.

By (2) $\left(x_{1} \cdot x_{2}\right) y$ is in $\mathfrak{N}$ and it follows from (2) that $\left[\left(x_{1} \cdot x_{2}\right) \cdot x_{3}\right] y$ $=\lambda_{3} x_{1} \cdot x_{2}+\left(x_{1} \cdot x_{2}\right) \cdot z_{3}+x_{3} \cdot\left[\lambda_{1} x_{2}+\lambda_{2} x_{1}+x_{1} \cdot z_{2}+x_{2} \cdot z_{1}-\left(x_{1} \cdot y\right) \cdot x_{2}-\left(x_{2} \cdot y\right)\right.$ $\left.\cdot x_{1}+\left(x_{1} \cdot x_{2}\right) \cdot y\right]-\left[\left(x_{1} \cdot x_{2}\right) \cdot y\right] \cdot x_{3}-\left(x_{3} \cdot y\right) \cdot\left(x_{1} \cdot x_{2}\right)+\left[\left(x_{1} \cdot x_{2}\right) \cdot x_{3}\right] \cdot y$. Without bothering to simplify interchange subscripts 1 and 3 to get $\left[x_{1} \cdot\left(x_{2} \cdot x_{3}\right)\right] y=\lambda_{1} x_{3} \cdot x_{2}+\left(x_{3} \cdot x_{2}\right) \cdot z_{1}+x_{1} \cdot\left[\lambda_{3} x_{2}+\lambda_{2} x_{3}+x_{3} \cdot z_{2}+x_{2} \cdot z_{3}-\left(x_{3} \cdot y\right)\right.$ $\cdot x_{2}-\left(x_{2} \cdot y\right) \cdot x_{3}-\left(x_{1} \cdot y\right) \cdot\left(x_{3} \cdot x_{2}\right)+\left[\left(x_{3} \cdot x_{2}\right) \cdot x_{1}\right] \cdot y$. Using the notation $(a, b, c)=(a \cdot b) \cdot c-a \cdot(b \cdot c)$ for the associator of $a, b, c$ in $\mathfrak{A}^{+}$we have, upon subtracting the second relation from the first, $\left(x_{1}, x_{2}, x_{3}\right) y$ $=\left(x_{1}, x_{2}, z_{3}\right)+\left(x_{1}, z_{2}, x_{3}\right)+\left(z_{1}, x_{2}, x_{3}\right)-\left(x_{1} \cdot y, x_{2}, x_{3}\right)-\left(x_{1}, x_{2} \cdot y, x_{3}\right)$ $+\left(x_{3} \cdot y, x_{2}, x_{1}\right)+\left(x_{1}, x_{2}, x_{3}\right) \cdot y$.

Now define the set $\mathfrak{B}$ to be the subspace of $\mathfrak{A}$ generated by the associators $(a, b, c)$ with $a, b, c$ in $\mathfrak{N}$ and using the product of $\mathfrak{N}^{+}$. We have proved the following lemma.

Lemma 1. Let $\mathfrak{A}$ be a nodal noncommutative Jordan algebra whose characteristic is not 2. Then $\mathfrak{B N} \subseteq \mathfrak{B}+\mathfrak{B} \cdot \mathfrak{N}$. Also $\mathfrak{N} \subseteq \subseteq \mathfrak{B}+\mathfrak{B} \cdot \mathfrak{N}$.

The last statement follows from the fact that if $b$ is in $\mathscr{B}, n$ in $\mathfrak{R}$, then $n b=2 b \cdot n-b n$.

Let $\mathfrak{C}_{0}=\mathfrak{B}, \quad \mathfrak{C}_{1}=\mathfrak{B}+\mathfrak{B} \cdot \mathfrak{N}=\mathfrak{C}_{0}+\mathfrak{C}_{0} \cdot \mathfrak{R}$, and in general $\mathfrak{C}_{i+1}=\mathfrak{C}_{i}$ $+\mathfrak{E}_{i} \cdot \mathfrak{R}$. Equivalently, $\mathfrak{S}_{i+1}=\mathfrak{C}_{i}+\mathfrak{B}\left(R_{\mathfrak{N}}^{+}\right)^{i+1}$.

Lemma 2. The product $(\mathfrak{B} \cdot \mathfrak{N}) \mathfrak{N} \subseteq \mathfrak{S}_{2}$ and $\mathfrak{N}(\mathfrak{B} \cdot \mathfrak{N}) \subseteq \mathfrak{\subseteq}_{2}$. It follows that $\mathfrak{S}_{1} \mathfrak{N} \subseteq \mathfrak{S}_{2}, \mathfrak{R} \mathfrak{S}_{1} \subseteq \mathfrak{\complement}_{2}$.

The proof follows from the flexible law as does (2) which was proved by Schafer. The linearized form of the flexible identity is

$$
(x y) z+(z y) x=x(y z)+z(y x) .
$$

Add $(y x) z+(y z) x$ to both sides of (3) to obtain the equivalent relation

$$
(x \cdot y) z+(y \cdot z) x=y z \cdot x+y x \cdot z .
$$


If $x$ is in $\mathfrak{B}, y, z$ in $\mathfrak{N}$, then $(y \cdot z) x$ is in $(\mathfrak{N} \cdot \mathfrak{N}) \mathfrak{B} \subseteq \mathfrak{N} \mathfrak{B}$. By Lemma 1 , $(y \cdot z) x$ is in $\mathfrak{C}_{1}$. The product $y z$ is in $\mathfrak{F} 1+\mathfrak{R}$ so $y z \cdot x$ is in $\mathfrak{B}+\mathfrak{N} \cdot \mathfrak{B}=\mathfrak{C}_{1}$. And $y x \cdot z$ is in $\mathfrak{N B} \cdot \mathfrak{R} \subseteq \mathfrak{C}_{2}$. Therefore, $(x \cdot y) z$ is in $\mathfrak{C}_{2}$ as desired.

LEMma 3. The product $\left[\mathfrak{B}\left(R_{\mathfrak{N}}^{+}\right)^{i}\right] \mathfrak{R} \subseteq \mathfrak{C}_{i+1}$ and $\mathfrak{N}\left[\mathfrak{B}\left(R_{\mathfrak{N}}^{+}\right)^{i}\right] \subseteq \mathfrak{C}_{i+1}$. Or , equivalently, $\mathfrak{C}_{i} \mathfrak{N} \subseteq \mathfrak{C}_{i+1}, \mathfrak{N} \mathfrak{C}_{i} \subseteq \mathfrak{C}_{i+1}$.

Assume that $\left[\mathfrak{B}\left(R_{\mathfrak{N}}^{+}\right)^{i-1}\right] \mathfrak{N}$ and $\mathfrak{N}\left[\mathfrak{B}\left(R_{\mathfrak{R}}^{+}\right)^{i-1}\right]$ are in $\mathfrak{C}_{\imath}$. Take $x$ in (4) to be in $\mathfrak{S}=\mathfrak{B}\left(R_{\mathfrak{R}}^{+}\right)^{i-1}$, and $y, z$ to be in $\mathfrak{R}$. Then $(y \cdot z) x$ is in $\mathfrak{N S} \subseteq \mathfrak{C}_{i}, y z \cdot x$ is in $\mathfrak{S}+\mathfrak{R} \cdot \mathfrak{S} \subseteq \mathfrak{C}_{i}$, and $y x \cdot z$ is in $(\mathfrak{R S}) \cdot \mathfrak{R} \subseteq \mathfrak{C}_{i+1}$. Thus $(x \cdot y) z$ is in $\mathfrak{C}_{i+1}$.

Lemma 4. There exists a positive integer $k$ such that $\mathfrak{\complement}_{k}=\mathfrak{S}_{k+1}$ and $\mathfrak{\mathfrak { S }}_{k}$ is an ideal of $\mathfrak{A}$.

The set $\mathfrak{B}$ is contained in $\mathfrak{N}$. Since $\mathfrak{H}^{+}$is a Jordan algebra, $\mathfrak{N}^{+}$is nilpotent. Consequently, $\mathfrak{B}\left(R_{\mathfrak{N}}^{+}\right)^{k+1}=0$ for some $k$. For this $k, \mathfrak{E}_{k}$ $=\mathfrak{C}_{k+1}$. By Lemma $3 \mathfrak{C}_{k} \mathfrak{N} \subseteq \mathfrak{C}_{k+1}=\mathfrak{C}_{k}$ and $\mathfrak{N} \mathfrak{C}_{k} \subseteq \mathfrak{C}_{k}$.

The ideal $\mathfrak{E}_{k}$ is contained in $\mathfrak{N} \cdot \mathfrak{N} \cdot \mathfrak{N}=\mathfrak{N}_{3}$. Since $\mathfrak{N}^{+}$is a subalgebra of $\mathfrak{A}^{+}, \mathfrak{N}_{3} \subset \mathfrak{R}$ and so $\mathfrak{E}_{k} \subset \mathfrak{N}$. If $\mathfrak{A}$ is a simple algebra, $\mathfrak{夭}_{k}=0$ and thus $\mathfrak{B}=0$. This says that every associator in $\mathfrak{N}^{+}$is zero. Now if $a, b, c$ are any elements in $\mathfrak{A}, a=\alpha 1+x, b=\beta 1+y, c=\gamma 1+z$ with $x, y, z$ in $\mathfrak{N}$. Then $(a \cdot b) \cdot c-a \cdot(b \cdot c)=(x \cdot y) \cdot z-x \cdot(y \cdot z)$ so every associator in $\mathfrak{Q}^{+}$is an associator in $\mathfrak{R}^{+}$. This completes the proof of the theorem.

Any ideal properly contained in $\mathfrak{A}$ is contained in $\mathfrak{N}$, hence is a nilideal and is contained in the radical of $\mathfrak{A}$. This implies the corollary which we state below.

COROLlary. Let $\mathfrak{A}$ be a semisimple nodal noncommutative Jordan algebra of characteristic $\neq 2$. Then $\mathfrak{A}$ is simple and $\mathfrak{A}^{+}$is associative.

\section{REFERENCES}

1. N. Jacobson, $A$ theorem on the structure of Jordan algebras, Proc. Nat. Acad. Sci. U.S.A. vol. 42 (1956) pp. 140-147.

2. L. A. Kokoris, Some nodal noncommutative Jordan algebras, Proc. Amer. Math. Soc. vol. 9 (1958) pp. 164-166.

3. - Nodal noncommutative Jordan algebras, Canadian J. Math. (to appear).

4. R. D. Schafer, On noncommutative Jordan algebras, Proc. Amer. Math. Soc. vol. 9 (1958) pp. 110-117.

WASHINGTON UNIVERSITY 